\title{
Mediterranean spotted fever and encephalitis: a case report and review of the literature
}

\author{
Vitor Duque • Conceição Ventura • Diana Seixas • \\ Arnaldo Barai · Nuno Mendonça · Joana Martins • \\ Saraiva da Cunha $\cdot$ António Meliço-Silvestre
}

Received: 6 May 2011/ Accepted: 10 August 2011

(C) Japanese Society of Chemotherapy and The Japanese Association for Infectious Diseases 2011

\begin{abstract}
Mediterranean spotted fever (MSF) is a disease caused by Rickettsia conorii and transmitted by the brown dog tick Rhipicephalus sanguineus. It is widely distributed through southern Europe, Africa, and the Middle East. It is an emerging or a reemerging disease in some regions. Countries of the Mediterranean basin, such as Portugal, have noticed an increased incidence of MSF over the past 10 years. It was believed that MSF was a benign disease associated with a mortality rate of $1-3 \%$ before the antimicrobial drug era. It was called benign summer typhus. Severe forms were described in 1981, and the mortality rate reached 32\% in Portugal in 1997. However, neurological manifestations associated with brain lesions are a
\end{abstract}

V. Duque $(\varangle) \cdot$ C. Ventura $\cdot$ D. Seixas $\cdot$ S. da Cunha

Serviço de Doenças Infecciosas, Hospitais da Universidade de Coimbra, EPE, Avenida Bissaya Barreto e Praceta Prof. Mota Pinto, 3000-075 Coimbra, Portugal

e-mail: duque.vitor@gmail.com

V. Duque $\cdot$ S. da Cunha $\cdot$ A. Meliço-Silvestre

Faculty of Medicine, University of Coimbra, Coimbra, Portugal

\section{A. Barai}

Serviço de Medicina Interna, Hospital Sousa Martins, EPE, Guarda, Portugal

N. Mendonça

Serviço de Neurologia, Hospitais da Universidade de Coimbra, EPE, Coimbra, Portugal

\section{J. Martins}

Serviço de Imagiologia, Hospitais da Universidade de Coimbra, EPE, Coimbra, Portugal

A. Meliço-Silvestre

AGI II, Hospitais da Universidade de Coimbra, EPE, Coimbra, Portugal rare event. We describe the case of a man with fever, maculopapular rash, a black spot, and hemisensory loss including the face on the left side of the body with brain lesions in the imaging studies.

Keywords Mediterranean spotted fever - Encephalitis · Brain lesions $\cdot$ Rickettsia conorii $\cdot$ Hemisensory loss

\section{Introduction}

Mediterranean spotted fever, also known in the medical literature as Boutonneuse fever and Marseilles fever [1], is an emerging zoonosis associated with Rickettsia conorii infection, an obligate intracellular, gram-negative bacterium. It is a member of the spotted fever group of rickettsiae and is widely distributed throughout southern Europe, Africa, and the Middle East.

The major ecologic niche of the rickettsiae affecting man is the brown dog tick, Ripicephalus sanguineus [2]. Rickettsiae are maintained in ticks through transtadial and transovarial transmission. Other ticks have been reported to harbor Rickettsia conorii, but Ripicephalus sanguineus is the only recognized tick vector identified in Portugal [3].

Most cases occur during the summer (87\%), especially during the period from July to September [4].

MSF is usually a benign self-limited exanthematic febrile disease. The case fatality rate is $3-7 \%$ among hospitalized patients. However, higher rates have been observed (32\% in 1997, in Portugal) [5].

Advanced age, chronic alcoholism, immunocompromised status, glucose-6-phosphate dehydrogenase deficiency, prior prescription of inappropriate antimicrobial therapy, delay in treatment, and diabetes are risk factors for severe MSF and therefore have been associated with more 
severe disease wherein a multiple-organ dysfunction syndrome can lead to a fatal outcome $[5,6]$.

Serological tests are the easiest methods to perform the diagnosis on a routine basis, and microimmunofluorescence is widely accepted as the reference method allowing the detection of $\operatorname{IgM}$ and $\operatorname{IgG}$ in acute and convalescent sera. Both IgM and IgG can be detected 7-15 days after the onset of the disease [7].

Doxycycline ( $200 \mathrm{mg} /$ day) is the drug of choice for the treatment of MSF for 5-7 days. Chloramphenicol $(50-75 \mathrm{mg} / \mathrm{kg} /$ day), given in four divided doses for $7-10$ days, and tetracycline $(25-50 \mathrm{mg} / \mathrm{kg} /$ day $)$, are alternatives to doxycycline treatment.

\section{Case report}

A 59-year-old Caucasian man was admitted to the infectious diseases clinic of the University Hospital of Coimbra on 25 September 2010 because of MSF with neurological complications.

The patient was resident in a rural area; he had two dogs protected from ticks by repellents.

On 5 September 2010, he noticed a bite on the left thigh and at the end of the day a small sore on the anterior thigh region could be seen.

On 13 September 2010, he started having a fever that reached $40^{\circ} \mathrm{C}$, headaches, myalgia, and abnormal sensations on the left side of the body.

He was observed by a physician, who noticed the presence of a maculopapular rash on the trunk and an eschar on the left anterior thigh region, and confirmed the presence of hemisensory loss on the left side of the body, including the face.

Treatment was started with doxycycline $100 \mathrm{mg}$ per os twice a day, which was maintained for 7 days at home.

The patient felt better when the fever abated 2 days later. Eight days later he was referred to our infectious diseases service.

The physical examination revealed a maculopapular rash over the trunk, sparing palms and soles. A dark brown eschar $1 \mathrm{~cm}$ in diameter was found on his left anterior thigh region (Fig. 1).

The neurological examination showed a hemisensory loss including the face on the left.

A brain computed tomography (CT) scan showed hypodensities in the white matter of the frontal lobes and one lesion in close proximity to the atrium of the left lateral ventricle, without mass effect or contrast enhancement.

Brain magnetic resonance imaging (MRI) showed multiple, non-contrast-enhanced, periventricular, white matter lesions hyperintense on $\mathrm{T}_{2}$-weighted sequences and isointense on $\mathrm{T}_{1}$ (Fig. 2a).

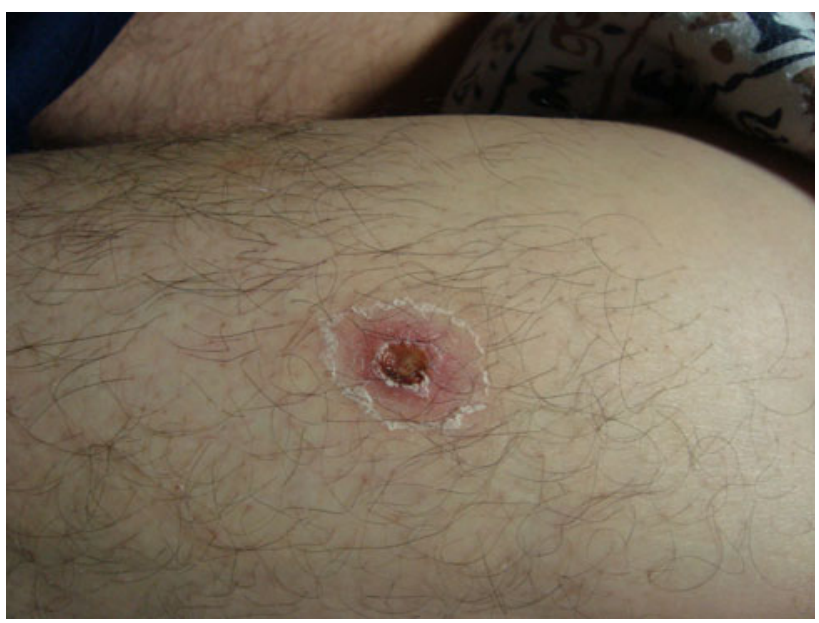

Fig. 1 Dark brown inoculation eschar (tache noir or black spot) in the left anterior thigh region, observed 20 days after the tick bite

A contrast-enhanced lesion in the right thalamus was observed, suggesting a recent vascular origin (Fig. 2b, c).

A lumbar puncture was performed. Cerebrospinal fluid (CSF) showed 2,000 red blood cells/ml with a normal content of leukocytes, slightly elevated protein content (49 $\mathrm{mg} / \mathrm{dl})$, and a normal glucose level.

Routine microbiological studies and polymerase chain reaction for herpes simplex virus were negative in the CSF. Serology for enteroviruses, arboviruses, brucellosis, syphilis, and human immunodeficiency virus (HIV)-1/2 was negative. An indirect immunofluorescent antibody test was performed at admission, showing elevated $\operatorname{IgM}$ and $\operatorname{IgG}$ titers to Rickettsia conorii (1:2,048 and 1:1,280, respectively).

Treatment was (re)started with doxycycline with a loading dose of $200 \mathrm{mg}$ intravenously every $12 \mathrm{~h}$ for the first 4 days and maintained at $100 \mathrm{mg}$ intravenously every $12 \mathrm{~h}$ during the next 3 days.

Clinical improvement was progressive during treatment with recovery of sensation on the left hemibody, except on the left hand and chin area.

On 2 October 2010, the patient was discharged after 7 days of retreatment.

On 12 October 2010, a brain MRI was repeated, and a smaller non-contrast-enhanced right thalamic lesion was observed. There were no alterations in the other scattered white matter lesions.

\section{Discussion}

Only four cases of encephalitis clearly related to Rickettsia conorii infection with brain lesions in imaging studies were described in the literature (one child and three adults). Brain CT and MRI scans were performed in two cases, but 
Fig. 2 Brain magnetic resonance imaging (MRI) showed multiple periventricular white matter lesions hyperintense on $\mathrm{T}_{2}$-weighted sequences and isointense on $\mathrm{T}_{1}(\mathbf{a})$. There was no contrast enhancement, suggesting sequelae of vascular origin. A right thalamus lesion was also observed (b) with contrast enhancement (c), suggesting a recent vascular origin. Edema, mass effect, or displacement of normal structures was not observed

lesions were only observed in the MRI scans whereas the CT scans were normal [8].

The other two patients had only brain lesions observed in brain CT scans because no brain MRI scans were performed.

Of the three adults, all over the age of 50 years, two recovered with severe neurological sequelae and only one survived without sequelae [8].

We describe the third case of severe MSF encephalitis in an adult with documented brain lesions and major neurological involvement who survived with neurological sequelae, despite appropriate treatment. This is also the first case with lesions documented in both CT and MRI scans.

The target cell for pathogenic rickettsiae is the vascular endothelial cell, which it invades and where it multiplies. The result is a widespread vasculitis of capillaries, arterioles, and small arteries that correlate with the presence of Rickettsia conorii [9].

Brain lesions consist of a mononuclear leukocyte infiltration of the blood vessel wall and perivascular space [9], but perivascular lymphohistiocytic infiltrates [1] and petechial brain hemorrhage have also been observed.

The brain MRI showed that our patient had vasculitic lesions, multiple periventricular white matter lesions without contrast enhancement interpreted as sequelae, and only one contrast-enhanced lesion in the right thalamus suggesting a recent vascular origin and interpreted as an active lesion (Fig. 2).

The right thalamus lesion was observed after 7 days of standard treatment with oral doxycycline, suggesting that the standard treatment is not sufficient to sterilize all the vasculitic lesions related to MSF.

The neurological picture associated with the right thalamus lesion improved only after a second course of intravenous doxycycline over another 7 days.

Penetration of doxycycline in the central nervous system could be classified as moderate, reaching a mean level in the CSF that is $26 \%$ of the simultaneous drug level in serum [10].

It has been described that standard orally administered doxycycline seems to give sufficient concentrations in serum and CSF for the treatment of MSF encephalitis, but a higher concentration can be reached when a dose double that of the standard dose is administered [11].
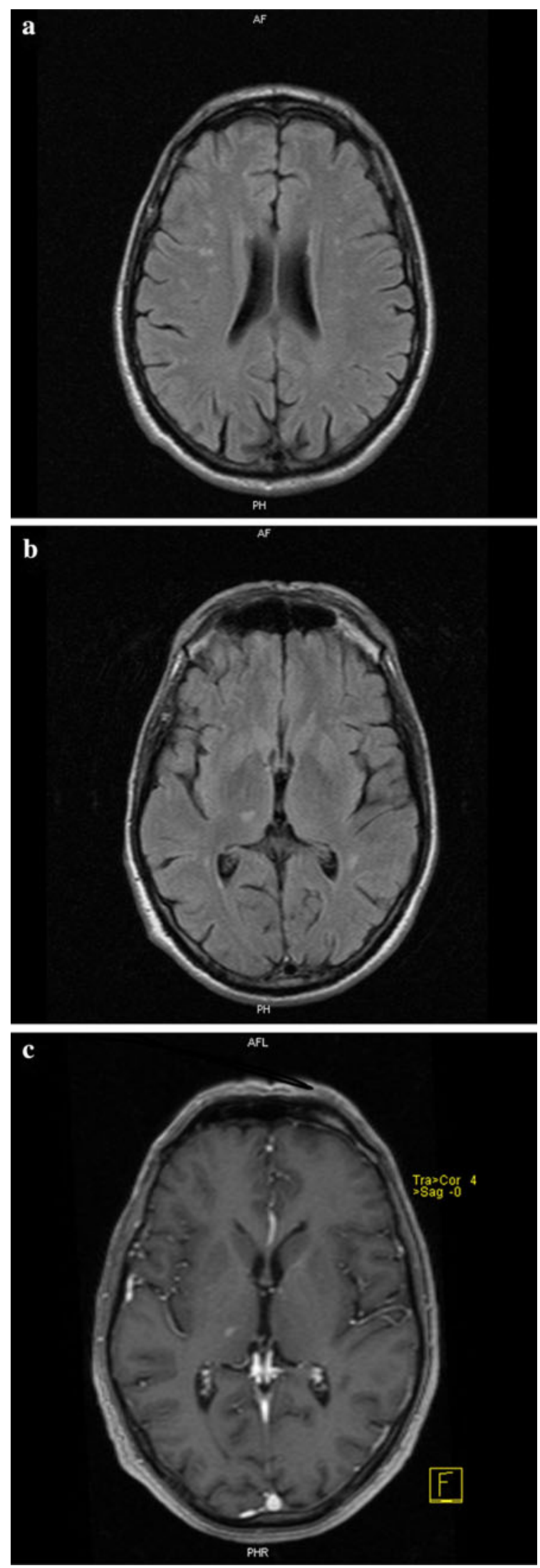
The case we described here is in agreement with the need for higher doses of doxycycline to treat MSF encephalitis.

We used a second course of intravenous doxycycline with double doses during the first 4 days followed by 3 days of standard dose.

The neurological picture of our patient improved after resuming therapy and was associated with a reduction in the right thalamic lesion, as observed on a second brain MRI (not shown).

The improvement observed, although not complete, could be explained because the intravenous route could eliminate the problem of the irregular absorption of doxycycline by the gastrointestinal tract and because higher plasma and brain concentrations could be reached [10].

Intravenous formulation and longer courses of doxycycline were given to patients with nausea and vomiting and to those who were seriously ill [12], but no clear length of treatment is defined for the management of MSF-related encephalitis.

Rickettsiae can persist up to 36 days in ischemic tissues of amputated fingers after the beginning of treatment with oral tetracycline. Also, vasculitis is the result of direct vascular injury in the brain [1].

Brain vasculitis may harbor viable rickettsiae for a similarly long time. In our case, first standard treatment with oral doxycycline was associated with clinical improvement of the peripheral manifestations of MSF, but no improvement was observed in the neurological manifestations. However, a clear clinical response to treatment was observed during the second course of intravenous treatment, which was documented with a reduction in the size of the right thalamic lesion responsible for the neurological picture of our patient.

Other lesions did not improve with treatment, but imaging suggested that they were not of recent vascular origin. No cause other than rickettsial infection explained their origin.

We conclude that uncomplicated MSF can be treated successfully with oral doxycycline but, in our case, suggest that patients with MSF and neurological manifestations should be treated with doxycycline early and by the intravenous route for at least 15 days.

\section{References}

1. Walker DH, Herrero-Herrero JI, Ruiz-Beltrán R, Bullón-Sopelana A, Ramos-Hidalgo A. The pathology of fatal Mediterranean spotted fever. Am J Clin Pathol. 1987;87:669-72.

2. Rovery C, Brouqui P, Raoult D. Questions on Mediterranean spotted fever a century after its discovery. Emerg Infect Dis. 2008;14:1360-7.

3. de Sousa R, Santos-Silva M, Santos AS, Barros SC, Torgal J, Walker DH, et al. Rickettsia conorii Israeli tick typhus strain isolated from Rhipicephalus sanguineus ticks in Portugal. Vector Borne Zoonotic Dis. 2007;7:444-7.

4. de Sousa R, Ismail N, Dória-Nóbrega S, Costa P, Abreu T, França $A$, et al. The presence of eschars, but not greater severity, in Portuguese patients infected with Israeli spotted fever. Ann N Y Acad Sci. 2005;1063:197-202.

5. Sousa R, França A, Dória Nòbrega S, Belo A, Amaro M, Abreu $\mathrm{T}$, et al. Host- and microbe-related risk factors for and pathophysiology of fatal Rickettsia conorii infection in Portuguese patients. J Infect Dis. 2008;198:576-85.

6. Parola P, Paddock CD, Raoult D. Tick-borne rickettsioses around the world: emerging diseases challenging old concepts. Clin Microbiol Rev. 2005;18:719-56.

7. Brouqui P, Bacellar F, Baranton G, Birtles RJ, Bjoërsdorff A, Blanco JR, et al. Guidelines for the diagnosis of tick-borne bacterial diseases in Europe. Clin Microbiol Infect. 2004;10: 1108-32.

8. Aliaga L, Sánchez-Blázquez P, Rodríguez-Granger J, Sampedro A, Orozco M, Pastor J. Mediterranean spotted fever with encephalitis. J Med Microbiol. 2009;58:521-5.

9. Walker DH, Gear JH. Correlation of the distribution of Rickettsia conorii, microscopic lesions, and clinical features in South African tick bite fever. Am J Trop Med Hyg. 1985;34:361-71.

10. Yim CW, Flynn NM, Fitzgerald FT. Penetration of oral doxycycline into the cerebrospinal fluid of patients with latent or neurosyphilis. Antimicrob Agents Chemother. 1985;28:347-8.

11. Dotevall L, Hagberg L. Penetration of doxycycline into cerebrospinal fluid in patients treated for suspected Lyme neuroborreliosis. Antimicrob Agents Chemother. 1989;33:1078-80.

12. Tunkel AR, Glaser CA, Bloch KC, Sejvar JJ, Marra CM, Roos $\mathrm{KL}$, et al. The management of encephalitis: clinical practice guidelines by the Infectious Diseases Society of America. Clin Infect Dis. 2008;47:303-27. 